\title{
The Correlation Between Wall Shear Stress and Plaque Composition in Advanced Human Carotid Atherosclerosis
}

\author{
A. M. Moerman ${ }^{1}$, S. Korteland ${ }^{1}$, K. Dilba ${ }^{1}$, K. van Gaalen ${ }^{1}$, D. H. J. Poot ${ }^{2}$, A. van Der Lugt ${ }^{2}$, \\ H. J. M. Verhagen ${ }^{3}$, J. J. Wentzel ${ }^{1}$, A. F. W. van Der Steen ${ }^{1}$, F. J. H. Gijsen ${ }^{1,4}$ and \\ K. Van der Heiden ${ }^{1 *}$ \\ ${ }^{1}$ Department of Biomedical Engineering, Thorax Center, Erasmus MC, Rotterdam, Netherlands, ${ }^{2}$ Department of Radiology and \\ Nuclear Medicine, Erasmus MC, Rotterdam, Netherlands, ${ }^{3}$ Department of Vascular Surgery, Erasmus MC, Rotterdam, \\ Netherlands, ${ }^{4}$ Department of Biomedical Engineering, Delft University of Technology, Delft, Netherlands
}

\section{OPEN ACCESS}

Edited by:

Der-Cherng Tarng,

Taipei Veterans General Hospital,

Taiwan

Reviewed by: Dalin Tang,

Worcester Polytechnic Institute,

United States

Aike Qiao,

Beijing University of Technology,

China

*Correspondence:

K. Van der Heiden

K.vanderheiden@erasmusmc.nI

Specialty section:

This article was submitted to Biomechanics,

a section of the journal

Frontiers in Bioengineering and Biotechnology

Received: 03 December 2021 Accepted: 29 December 2021

Published: 28 January 2022

Citation:

Moerman AM, Korteland S, Dilba K,

van Gaalen K, Poot DHJ,

van Der Lugt A, Verhagen HJ,

Wentzel J, van Der Steen AFW, Gijsen FJ and Van der Heiden K (2022)

The Correlation Between Wall Shear

Stress and Plaque Composition in

Advanced Human

Carotid Atherosclerosis.

Front. Bioeng. Biotechnol. 9:828577.

doi: 10.3389/fbioe.2021.828577
The role of wall shear stress (WSS) in atherosclerotic plaque development is evident, but the relation between WSS and plaque composition in advanced atherosclerosis, potentially resulting in plaque destabilization, is a topic of discussion. Using our previously developed image registration pipeline, we investigated the relation between two WSS metrics, time-averaged WSS (TAWSS) and the oscillatory shear index (OSI), and the local histologically determined plaque composition in a set of advanced human carotid plaques. Our dataset of 11 carotid endarterectomy samples yielded 87 histological crosssections, which yielded 511 radial bins for analysis. Both TAWSS and OSI values were subdivided into patient-specific low, mid, and high tertiles. This cross-sectional study shows that necrotic core (NC) size and macrophage area are significantly larger in areas exposed to high TAWSS or low OSI. Local TAWSS and OSI tertile values were generally inversely related, as described in the literature, but other combinations were also found. Investigating the relation between plaque vulnerability features and different combinations of TAWSS and OSI tertile values revealed a significantly larger cap thickness in areas exposed to both low TAWSS and low OSI. In conclusion, our study confirmed previous findings, correlating high TAWSS to larger macrophage areas and necrotic core sizes. In addition, our study demonstrated new relations, correlating low OSI to larger macrophage areas, and a combination of low TAWSS and low OSI to larger cap thickness.

Keywords: atherosclerosis, wall shear stress, oscillatory shear index, vulnerable plaque, plaque composition, MRI, image registration pipeline

\section{INTRODUCTION}

Atherosclerosis is a gradually progressing disease of the arteries characterized by vessel wall thickening due to the accumulation of lipids and inflammatory cells. This is referred to as plaque formation. Atherosclerosis is a multifactorial disease that can be aggravated not only by lifestyle factors, such as high caloric diet, physical inactivity, and smoking, but also by genetic factors. The initiation of plaque formation, however, has been strongly linked to a hemodynamic parameter: wall shear stress (WSS). WSS is the frictional force exerted by flowing blood on the vessel wall. 
Atherosclerotic plaques form at predetermined locations where WSS is low and/or oscillatory, which increases endothelial cell permeability and subsequent retention of lipoproteins (Lusis, 2000) and regulates pro-inflammatory signaling pathways in the endothelium, resulting in an increased influx of inflammatory cells (Gimbrone and García-Cardeña, 2013). The causal role of WSS in plaque initiation is evident (Kwak et al., 2014; Cunningham and Gotlieb, 2005); however, the influence of WSS on plaque progression is less clear. Initial plaque growth is generally accompanied by outward remodeling and preservation of low and/or oscillatory WSS levels. With disease progression, however, plaques will start to intrude the lumen, thereby affecting local hemodynamics. Upstream and at the throat of the plaque, WSS levels are high, while low WSS is found downstream (Slager et al., 2005). Based on their composition and the resulting risk of rupture, plaques are classified as stable or vulnerable. Vulnerable plaques are characterized by a thin cap, covering a large necrotic core (NC), and often present with high inflammatory activity and decreased smooth muscle cell content (Schaar et al., 2004). Intraplaque hemorrhage (IPH) can also be present (Virmani et al., 2000). The morphology and composition of advanced plaques were shown to be inhomogenous, both in the axial and in the circumferential direction (Richardson et al., 1989; Dirksen et al., 1998; Burke et al., 1999; Wentzel et al., 2003; Cicha et al., 2011), which could indicate a relation between local hemodynamics and plaque composition. In addition, plaque rupture is most often encountered at the high WSS-exposed upstream site (de Weert et al., 2009). The interplay between WSS and plaque composition is a subject of debate: both low and high WSS have been linked to features of plaque vulnerability (Lovett and Rothwell, 2003; Cicha et al., 2011; Eshtehardi et al., 2012; Wentzel et al., 2012; Vergallo et al., 2014; Tuenter et al., 2016; Yamamoto et al., 2019). However, these studies differ in imaging modality used for assessing plaque composition, complicating one-to-one comparison of their results. In addition, studies of the carotid artery are often based on assumed WSS levels (Dirksen et al., 1998; Lovett and Rothwell, 2003; Fagerberg et al., 2010) or used animal models, which are not truly representative (Winkel et al., 2015; Daugherty et al., 2017). Since histology is the gold standard for the assessment of human plaque composition in high resolution, we previously developed a framework for accurate registration of MRI-derived WSS patterns to histological cross-sections (Moerman et al., 2019). In this study, we investigated the correlation between WSS and histologically identified components of plaque vulnerability in a dataset of advanced human carotid atherosclerotic plaques.

\section{METHODS}

\section{MR Imaging, Tissue Collection, and Histological Processing}

MR imaging, tissue collection, tissue processing, and image registration procedures have been described in detail elsewhere (Moerman et al., 2019). In short, we imaged the carotid bifurcations of 11 patients scheduled for carotid endarterectomy (CEA) surgery in a 3.0-T MRI scanner (GE
Healthcare, Milwaukee, United States). The MRI protocol consisted of a 3D black-blood fast spin-echo (3D-BB-FSE) sequence with variable flip angles (TR/TE: 1000/16 ms; FOV: $15 \mathrm{~cm}$; slice thickness: $0.8 \mathrm{~mm}$; matrix: $160 \times 160$; number of excitations 1; scan time: $190 \mathrm{~s}$ ), which was optimized for visualizing lumen and outer wall geometry. CEA specimens were collected within $30 \mathrm{~min}$ after surgical resection, snapfrozen in liquid nitrogen, and stored at $-80^{\circ} \mathrm{C}$ until ex vivo scans and histology were performed. Upon processing, CEA specimens were thawed, fixed in $4 \%$ formaldehyde, and immersed in PBS. Ex vivo MRI scans (T2w fast recovery FSE (frFSE); TR/TE: 2,500/66 ms; in-plane resolution: $0.1 \times 0.1 \mathrm{~mm}$; slice thickness: $0.5 \mathrm{~mm}$; matrix: $256 \times 256$; scan time: $\sim 20 \mathrm{~min}$; number of slices: 66$)$ were performed using a 7.0-T MRI scanner (7.0T Discovery MR901, GE Healthcare, Milwaukee, United States).

CEA specimens were decalcified and cut into $1 \mathrm{~mm}$ thick consecutive axial cross-sections. "En face photos" were taken (IXUS 60, Canon, Tokyo, Japan) of the proximal side of each cross-section. The en face photos contained landmarks to facilitate registration of each en face photo to the photo of the adjacent cross-section, enabling reconstruction of a $3 \mathrm{D}$ stack of en face photos. After photographing, the 1-mm-thick axial crosssections were embedded in paraffin. Each paraffin block was cut into consecutive $5 \mu \mathrm{m}$ thick sections, which were processed for a series of histochemical staining procedures, that is, Miller's elastic stain, hematoxylin-eosin, Martius scarlet blue, and Picrosirius red, as well as two immunohistological staining procedures for macrophages (CD68, Abcam, United Kingdom) and endothelial cells (CD31, Abcam, United Kingdom). For each tissue section, compositional characteristics of plaque vulnerability, that is, necrotic core (NC) and the IPH-associated protein fibrin, were delineated in a segmentation image (BioPix iQ3.2). This segmentation was based on the total set of histochemical stains. Based on the immunohistological staining for CD68, we made segmentation masks of the CD68-positive pixels by applying a color deconvolution filter in Fiji (Ruifrok and Johnston, 2001; Schindelin et al., 2012). Tissue areas containing artifacts and NC led to false-positive results and were removed from CD68-positive pixel selection.

\section{Computational Fluid Dynamics for Wall Shear Stress Calculation}

Lumen contours of the stenosed bifurcations were delineated on the in vivo MRI scan using ITK-snap (Yushkevich et al., 2006) and exported as surface geometry VMTK (Antiga et al., 2008) was used for surface smoothing and centerline calculation. Clipping of inlet and outlets normal to the centerline was performed in ICEM (ANSYS ICEM 17.1, United States), and flow extensions were applied on the inlet and outlets using the VMTK. The length of the added flow extensions was five times the radius of the inlet or outlets. Subsequently, ICEM was used to generate a volume mesh containing on average 7 million elements. The volume mesh consisted of tetrahedral elements with five layers of prism elements at the wall. On the inlet of the common carotid artery (CCA), we applied a transient flow velocity profile using 
the theory of Womersley (Xu et al., 2018). This transient profile was based on the average flow waveform over one heart cycle and the heart rate reported by Lee et al. (2008). For each patient, the transient flow waveform was scaled to obtain an average WSS in the common carotid artery of 0.9 Pa (Lee et al., 2009) (Gnasso et al., 1997). As outlet boundary condition, the relative outflow to the internal carotid artery (ICA) and external carotid artery (ECA) was defined based on the stenosis degree (Groen et al., 2010). Blood density was set to $1060 \mathrm{~kg} / \mathrm{m}^{3}$, and non-Newtonian fluid behavior was mimicked by the Carreau-Yasuda model using the parameters reported by Seo et al. (2005). The Navier-Stokes equations were solved numerically (ANSYS Fluent 17.1, United States) (convergence criteria: $1 \mathrm{E}-4$ for continuity residual; $1 \mathrm{E}-5$ for $\mathrm{x}$-velocity, $\mathrm{y}$-velocity, and $\mathrm{z}$-velocity residuals; time step was $0.004 \mathrm{~s}$ ) over 2 heart cycles. Timedependent time-averaged wall shear stress (TAWSS) and oscillatory shear index (OSI) (Ku et al., 1985) were computed over the second heart cycle to account for initialization effects.

\section{Image Registration of MRI, CFD, and Histology}

Image registration was performed according to the methods we described previously (Moerman et al., 2019). In short, via a series of rigid and nonrigid image registrations and transformations, the in vivo MRI lumen and its corresponding WSS map were transformed consecutively to the ex vivo and the en face image domain. Histology images were axially stacked and registered to the en face domain as well, resulting in co-registration of in vivo MRI-derived WSS and histology.

\section{Analysis and Exclusion Criteria}

For a detailed description of data selection and analysis procedures, we refer to our previous publication (Moerman et al., 2019). In short, WSS patterns were axially averaged over $-0.3 \mathrm{~mm}$ to $+0.3 \mathrm{~mm}$ with respect to the axial location of the nearest histology section. Transversally, the WSS distribution over each lumen was discretized into eight radial bins (bin radius $45^{\circ}$ ). The points on the centerline of the transformed 3D WSS map were used as center points to create the radial bins. Plaque component measures were averaged in each radial bin. Per bin, we investigated the relation between local WSS and tissue composition of total intima depth. The dataset of $11 \mathrm{CEA}$ samples yielded 183 axial cross-sections, of which 87 (48\%) were included in the final analysis, yielding a total of 696 radial bins, of which $511(73 \%)$ were included in the final analysis.

We excluded data, both axial cross-sections and radial bins, based on a set of criteria. Axial cross-sections were excluded if A) a part of the excised CEA lumen showed severe and nonuniform deformation with respect to the rest of the lumen, for example, large lumen collapse in the ICA compared to the CCA. The applied nonrigid lumen registration algorithms act on the global $3 \mathrm{D}$ geometry and are not able to correct for local severe tissue deformations; thus, accurate registration was impaired in such cross-sections. B) Lumen diameter on in vivo MRI was $<3$ pixels and/or showed a very large axial gradient. As discussed previously (Moerman et al., 2019), the applicability of our method should be carefully assessed in these cases since WSS calculations in highly stenotic areas are very sensitive to minor variations in lumen size, and a small mismatch in registration accuracy in regions with large axial gradients has a relatively large effect on the relation found between WSS and plaque composition. C) Low signal-tonoise ratio of the in vivo MRI images, which impaired reliable lumen segmentation. The radial bins were excluded based on the presence of 1) histological processing artifacts, 2) a mismatch in lumen registration between en face and histology images, or 3) a mismatch in lumen registration between in vivo MRI and en face images (Moerman et al., 2019). For the included radial bins, we quantified registration performance by calculating the average Hausdorff distance (HD) and dice similarity coefficients (DSCs) between en face and histology lumen segmentations and en face and in vivo MRI lumen segmentations (Moerman et al., 2019).

\section{Statistical Analysis}

The range of absolute TAWSS and OSI values varied per artery. To define low, mid, and high WSS metric regions within each artery, patient-specific TAWSS and OSI tertiles were calculated. Statistical analysis was performed using a linear mixed effects model: WSS tertiles or OSI tertiles were set as a fixed factor and patient as a random factor. In addition, we tested the combination of WSS tertiles and OSI tertiles, including their interaction term, in one model to estimate the various parameters. Bonferroni correction was applied to adjust for multiple comparisons between WSS tertiles. The estimated means and standard error are reported. $P$-values $<0.05$ were considered statistically significant.

\section{RESULTS}

\section{Registration Performance}

The dataset of 11 CEA samples yielded 183 axial cross-sections, of which 87 (48\%) were included in the final analysis. Ninety-six axial cross-sections were excluded; the majority of exclusions (59) were due to severe nonuniform deformation in the excised tissue or processing artifacts. Twenty axial cross-sections were excluded because the in vivo lumen was highly stenotic or had a large axial gradient, impairing accurate lumen segmentation for CFD simulations. Seventeen cross-sections were excluded because of bad signal-to-noise ratio on in vivo MRI. The 87 included axial crosssections were divided into 8 radial bins, yielding a total of 696 radial bins, of which 511 (73\%) were included in the final analysis. The majority of excluded radial bins (111) presented with histological artifacts. After the exclusion procedure, registration accuracy of the remaining dataset was quantified by calculating the dice similarity coefficient (DSC) and Hausdorff distance (HD) between the lumen segmentations of the en face and histology cross-sections and between the en face and the transformed in vivo cross-sections. For the histology-to-en face registration, we found a mean HD of $0.55 \pm 0.07$ (SEM) mm and a mean DSC of $0.85 \pm 0.02$ (SEM). The registration performance between in vivo MRI and en face was slightly better: the mean HD was $0.36 \pm 0.06$ (SEM) $\mathrm{mm}$ and the mean DSC was $0.91 \pm 0.02$ (SEM). These values were in the order of magnitude of previously reported similarity indices, describing 


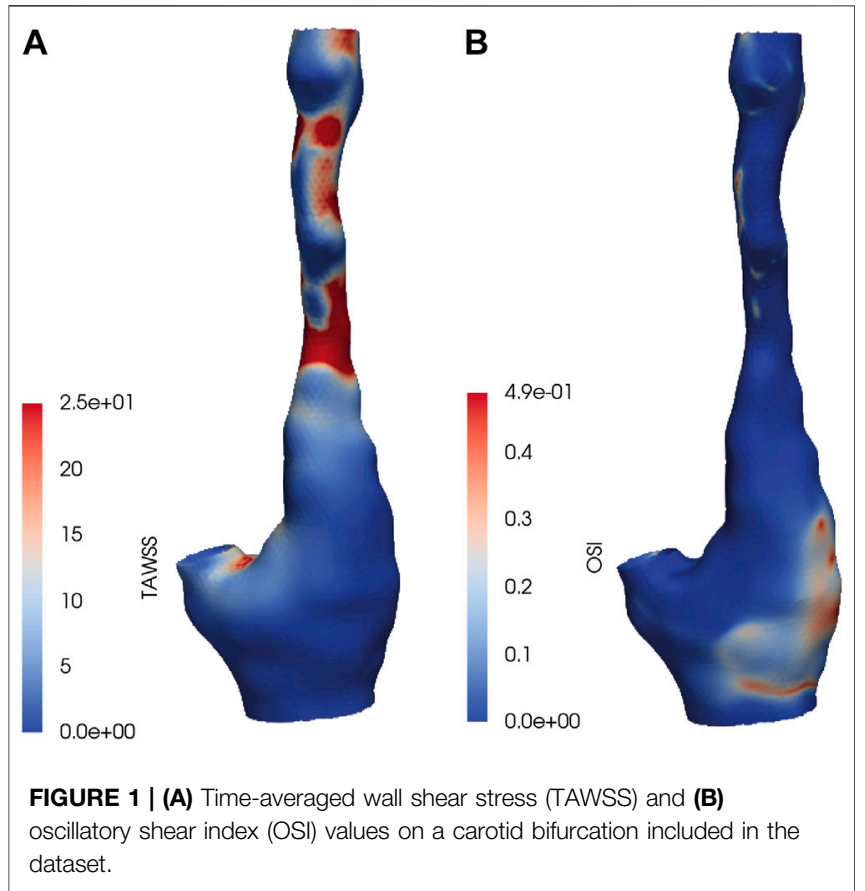

registration of images of CEA specimens by multiple imaging modalities (Boekhoven et al., 2013).

\section{Wall Shear Stress Patterns in Advanced Carotid Atherosclerosis}

In Figure 1 the TAWSS and OSI patterns on a carotid bifurcation are shown. In all included carotid arteries, relatively high TAWSS was mainly located at the flow divider, at the ECA inlet, and upstream to and at locations of the maximally narrowed lumen. Low TAWSS was mainly found in the proximal CCA, at the lateral side of the carotid bulb, and at sites of relative lumen dilation. High OSI was generally found at the lateral side of the carotid bulb and downstream of stenoses, while low OSI was seen in relatively straight arterial segments, such as the proximal CCA, and at sites of lumen narrowing. The range of absolute TAWSS and OSI values varied per artery. After registration of the WSS maps to histology, WSS values were axially and radially averaged. In Table 1 the patient-specific tertile boundaries and the ranges of average TAWSS and OSI values, after registration and averaging per bin, are reported. In Table 2, the co-occurrences of TAWSS and OSI tertile values are reported. An inverse relation between TAWSS and OSI tertile values was most frequently encountered, but other combinations were also found.

\section{Histologically Determined Plaque Composition in Advanced Carotid Atherosclerosis}

In Figure 2, a selection of representative histological crosssections is shown, along with the segmentation of lumen, fibrin, intima, NC, and macrophages. In general, histological cross-sections originating from the CCA showed a thickened intima with one or more NCs and small, elongated patches of macrophages. Both the NCs and the macrophages were generally located from the lumen up to a depth of half of the intima (Figure 2A). Relatively small fibrin areas could be observed as well. When moving from the proximal to distal region through the bulb, the plaque thickness varied in circumferential direction: plaque area and $\mathrm{NC}$ size were larger at the lateral side of the bifurcation (Figures 2B,C). Largest plaque area and eccentric plaque growth were observed in cross-sections harvested from the ICA (Figures 2D,E). Large NCs were present and showed large fibrin-positive areas. In these cross-sections, macrophages were generally distributed around the circumference of the lumen, were present in the cap, and were also seen deeper in the intima and at the edges of the NCs (Figure 2E). The amount of fibrous tissue was relatively low compared to proximal axial locations.

\section{Relation Between Wall Shear Stress Metrics and Plaque Composition}

For all included radial bins, we compared the average TAWSS and OSI levels, subdivided into patient-specific low, mid, and high

TABLE 1 | Patient-specific tertile boundaries and ranges of wall shear stress (WSS) metrics averaged on radial bins. TAWSS, time-averaged WSS; OSI, oscillatory shear index.

\begin{tabular}{|c|c|c|c|c|c|c|}
\hline Patient & $\begin{array}{c}\text { TAWSS tertile } \\
\text { boundary } \\
\text { low-mid [Pa] }\end{array}$ & $\begin{array}{l}\text { TAWSS tertile } \\
\text { boundary } \\
\text { mid-high } \\
\text { [Pa] }\end{array}$ & $\begin{array}{c}\text { Range } \\
\text { TAWSS-averaged } \\
\text { over bins } \\
{[\mathrm{Pa}]}\end{array}$ & $\begin{array}{c}\text { OSI tertile } \\
\text { boundary } \\
\text { low-mid [Pa] }\end{array}$ & $\begin{array}{l}\text { OSI tertile } \\
\text { boundary } \\
\text { mid-high } \\
\text { [Pa] }\end{array}$ & $\begin{array}{c}\text { Range } \\
\text { OSI-averaged } \\
\text { over bins } \\
\text { [Pa] }\end{array}$ \\
\hline 1 & 2.1 & 4.5 & $0.5-10.4$ & 0.003 & 0.010 & 0-0.092 \\
\hline 2 & 1.0 & 1.9 & $0.3-3.8$ & 0.004 & 0.018 & $0-0.386$ \\
\hline 3 & 1.5 & 2.0 & $1.0-3.3$ & 0.000 & 0.001 & 0-0.005 \\
\hline 4 & 1.3 & 2.0 & $0.5-3.5$ & 0.011 & 0.021 & $0-0.169$ \\
\hline 5 & 1.1 & 2.5 & $0.2-8.0$ & 0.001 & 0.014 & $0-0.093$ \\
\hline 6 & 1.2 & 2.8 & $0.5-34.8$ & 0.002 & 0.044 & $0-0.344$ \\
\hline 7 & 1.1 & 2.9 & $0.2-6.0$ & 0.002 & 0.008 & $0-0.111$ \\
\hline 8 & 0.6 & 1.5 & $0.2-5.8$ & 0.007 & 0.032 & $0-0.232$ \\
\hline 9 & 1.8 & 8.8 & $0.1-52.6$ & 0.000 & 0.013 & $0-0.308$ \\
\hline 10 & 1.7 & 2.7 & $1.0-11.1$ & 0.000 & 0.004 & $0-0.091$ \\
\hline 11 & 0.8 & 2.5 & $0.3-11.7$ & 0.004 & 0.031 & $0-0.156$ \\
\hline
\end{tabular}


TABLE 2 | Co-occurrence of TAWSS and OSI tertiles.

\begin{tabular}{lccc}
\hline & Low OSI & Mid OSI & High osı \\
\hline Low TAWSS & 11 & 38 & 120 \\
Mid TAWSS & 42 & 89 & 35 \\
High TAWSS & 116 & 39 & 14 \\
\hline
\end{tabular}

tertiles, to the composition of the underlying plaque (NC area, fibrin area, macrophage area, and cap thickness). In Figure 3, the relations between WSS metrics and plaque composition are visualized. When considering the plaque composition of the total intimal area per radial bin, we found significantly larger $\mathrm{NC}$ areas in plaque regions exposed to high TAWSS, compared to low TAWSS $(p=0.000)$ and mid TAWSS $(p=0.004)$. In addition, we found significantly larger $\mathrm{NC}$ areas in plaque regions exposed to low OSI, compared to high OSI $(p=0.000)$ and mid OSI $(p=$ $0.007)$. Regarding macrophage area, we found significantly larger macrophage areas in regions exposed to high TAWSS than in regions exposed to low TAWSS ( $p=0.007)$. In addition, we found significantly larger macrophage areas in plaque regions exposed to low OSI, compared to high OSI $(p=0.002)$ and mid OSI $(p=$ 0.002 ). When different combinations of TAWSS and OSI tertile values were analyzed, we found a significantly larger cap thickness in plaque regions exposed to both low TAWSS and low OSI, compared to low TAWSS and high OSI $(p=0.013)$. In the regions exposed to low OSI, a trend is observed for decreasing cap thickness with increasing TAWSS (low vs. mid TAWSS $p=$ 0.076 and low vs. high TAWSS $p=0.060$ ). No relations were found between the WSS metrics and fibrin area.

\section{DISCUSSION}

This was the first study that was able to make a direct comparison between local WSS metrics and histologically determined compositional characteristics of plaque vulnerability in a substantial number of human carotid plaques. The applied image registration pipeline allowed for a very accurate, localized assessment of this relation. We were able to take into account not just axial but also rotational matching of WSS and histology. Our cross-sectional analysis showed a relation between NC area and macrophage area with high TAWSS, as well as low OSI in advanced carotid atherosclerosis. Of these correlations, only a relation between presumed (because of the upstream location) high TAWSS and macrophage area was previously reported (Dirksen et al., 1998; Fagerberg et al., 2010). The relations between high TAWSS and cap thickness (Wentzel et al., 2013) in human coronaries and high TAWSS and IPH (Groen et al., 2007; Tuenter et al., 2016) in human carotids (Dirksen et al., 1998; Fagerberg et al., 2010), as previously reported, were not present in this dataset. When analyzing combinations of TAWSS and OSI tertile values, we found a significantly larger cap thickness in plaque regions exposed to

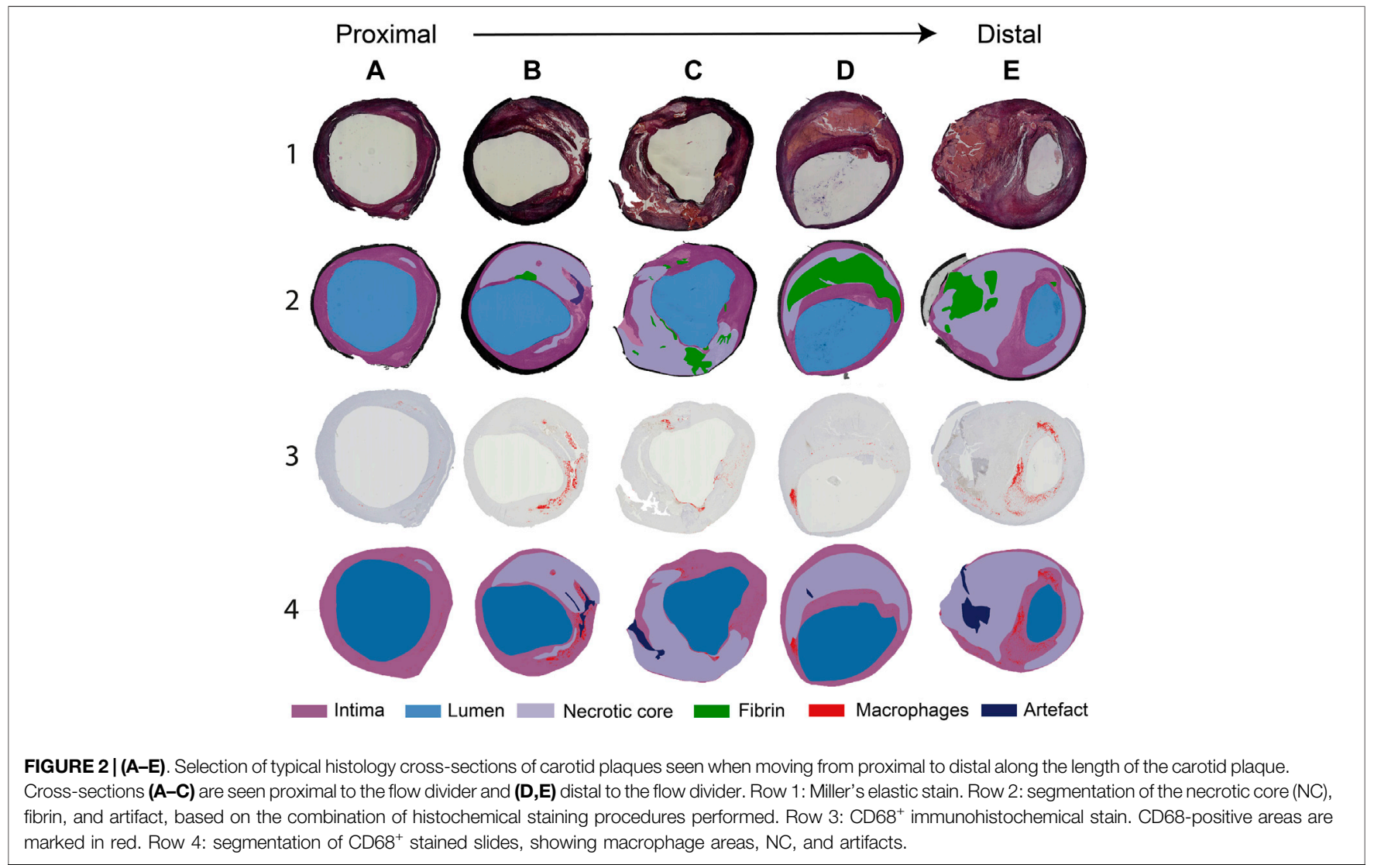


NC area

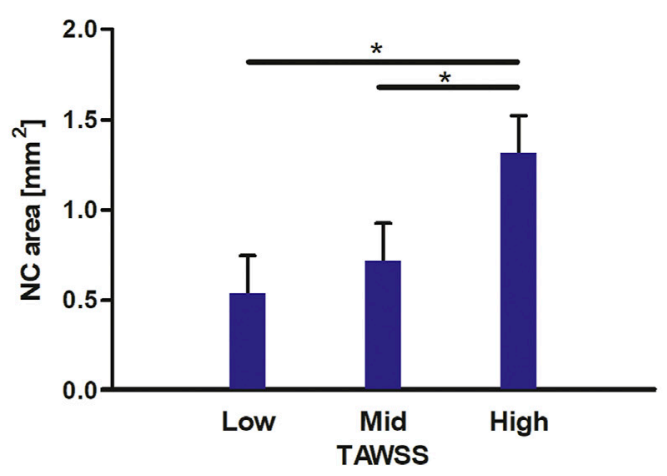

Macrophage area

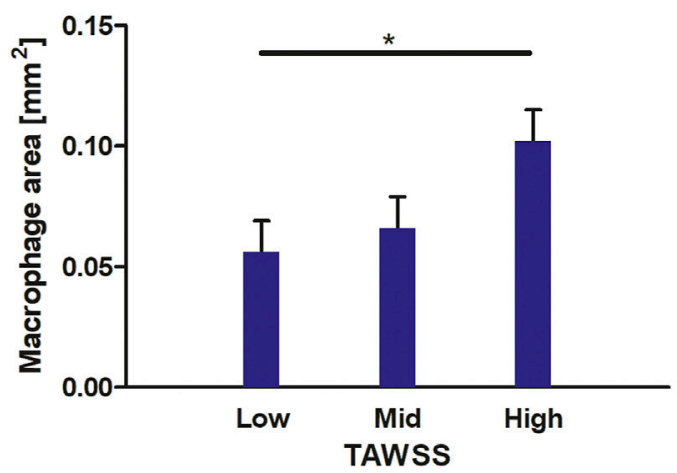

NC area

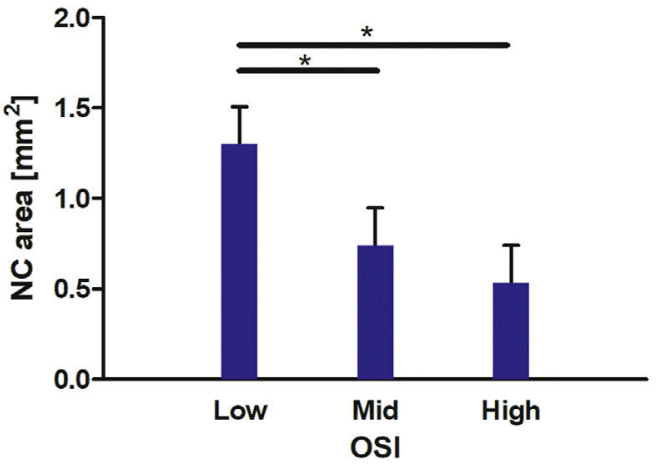

Macrophage area

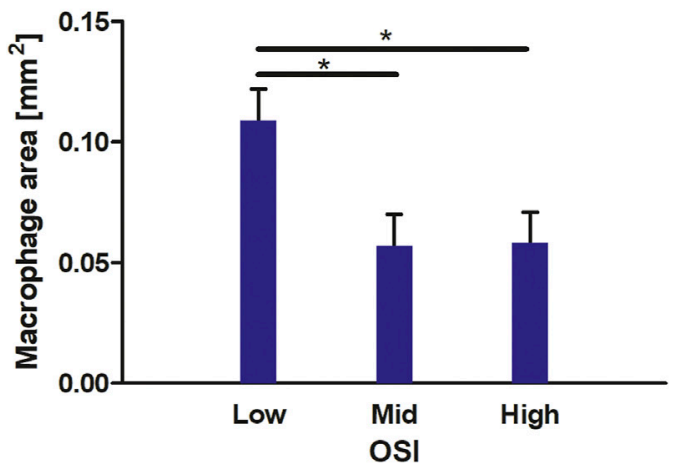

FIGURE 3 |Relations between WSS metrics and plaque compositional characteristics. In high TAWSS-exposed regions and low OSI-exposed regions, a larger NC area and macrophage area are found. At low TAWSS and low OSI, a larger cap thickness is found. Data are presented as estimated mean + standard error. ${ }^{*} p<0.05$.

both low TAWSS and low OSI, compared to low TAWSS and high OSI. The observed trend for decreasing cap thickness with increasing TAWSS in regions exposed to low OSI confirmed previous findings. ${ }^{12,20}$

\section{Wall Shear Stress Patterns in Advanced Carotid Atherosclerosis}

The TAWSS patterns observed in this set of carotid arteries were in line with findings previously described in the literature $(\mathrm{Ku}$ et al., 1985; Kaazempur-Mofrad et al., 2004; Ladisa et al., 2010; Gallo et al., 2016; van Ooij et al., 2018; Vamsi Krishna et al., 2020). That is, low TAWSS was present at the CCA, at the lateral side of the carotid bulb, while high TAWSS was mainly seen at the upstream side of lumen stenoses. Generally, an inverse relation between TAWSS and OSI was found, as described before (Gallo et al., 2016). However, a subset of radial bins showed other relations between these two WSS metrics. As inflow boundary conditions, we did not use measured patient-specific flow profiles but applied estimated patient-specific flow profiles, scaled by inlet diameter, as we previously found that the absolute WSS levels in highly stenotic carotid arteries were affected mostly by the patient-specific geometry rather than the flow (Groen et al., 2010). Yet, large variations in WSS and OSI values were still encountered between patients, which stresses the large influence of geometrical factors on WSS patterns. Because of the large variation in WSS ranges between patients, it would be impossible to draw conclusions on the relation between WSS and plaque composition by using absolute WSS values. Therefore, we calculated patient-specific WSS tertiles. The use of relative instead of absolute WSS levels has the additional advantage of diminishing the sensitivity of the CFD results to the approximated boundary conditions that were applied.

\section{Histologically Determined Plaque Composition in Advanced Carotid Atherosclerosis}

The histologically determined composition seen in this set of carotid plaques reflected histology results reported in the literature (Virmani et al., 2006b). That is, the greatest plaque cross-sectional area and smallest lumens were found in the ICA 
at cross-sections where the largest NCs were present, sometimes accompanied by fibrin-positivity, reflecting IPH. The majority of the excluded axial cross-sections and radial bins presented with processing artifacts, especially in the distal part of the plaque. The relatively "soft" necrotic and thrombotic tissue areas were more prone to deformation than tissue areas containing a higher amount of fibrous tissue. In addition, the tissue structure at areas that contained a high amount of calcium lost rigidity after decalcification, making these regions also susceptible to processing artifacts. The exclusion of these necrotic and calcified areas may have introduced a bias in our dataset toward moderately diseased tissue segments. The effect of this bias on the obtained results is unpredictable.

\section{Relation Between Wall Shear Stress Metrics and Plaque Composition}

Our study was designed to assess the correlations between WSS metrics and compositional features of plaque vulnerability in carotid plaques. We found relations between TAWSS and OSI with NC area, macrophage area, and cap thickness. Regarding NC area, we found that NCs were significantly larger at locations exposed to high TAWSS. Besides the existence of a direct correlation between TAWSS and NC size, this finding might be explained by the fact that progression of atherosclerosis is accompanied by plaque growth and subsequent lumen intrusion. This local lumen narrowing will affect TAWSS patterns, inducing a local high TAWSS at the upstream side and at the throat of the stenosis. In addition, plaque area has been shown to be correlated to NC size (Wentzel et al., 2013; Ahmadi et al., 2015), a finding that links the co-existence of high TAWSS and NC size. A positive correlation between TAWSS and NC has been reported before in both carotid plaques (Yang et al., 2010) and in coronary plaques (Samady et al., 2011; Eshtehardi et al., 2012; Wentzel et al., 2013; Park et al., 2016). Similarly, we also found significantly larger NCs at regions exposed to low OSI. The OSI value describes how much the WSS vector deviates from its average direction. Thus, low OSI, indicating a relative constant WSS direction, is mainly expected at regions with high flow velocity and high TAWSS. Although a large NC size has been shown to be associated with rupture (Virmani et al., 2006a; Falk et al., 2013), based on this crosssectional study, we cannot differentiate whether we are looking at a "true" relation between WSS-induced NC growth and vulnerability or simply at the effect of stenosis-induced altered hemodynamics. In addition to a larger NC area, we found significantly larger macrophage areas in plaque regions where OSI levels were low or TAWSS was high. Our findings confirmed the previously reported relation between presumed (because of the upstream location) high TAWSS and macrophage area (Dirksen et al., 1998; Fagerberg et al., 2010). To the best of our knowledge, we are the first to report a relation between low OSI and macrophage area. We found a new relation between cap thickness and WSS metrics when analyzing combinations of TAWSS and OSI tertile values. In plaque regions exposed to both low TAWSS and low OSI, cap thickness was significantly higher than in areas exposed to low TAWSS and high OSI. In addition, we observed a trend for decreasing cap thickness with increasing TAWSS in regions exposed to low OSI, confirming previous findings. ${ }^{12,20}$

\section{Implications of This Study}

The relation between WSS and plaque vulnerability has been a topic of discussion for many years. Finding a direct association between WSS and risk of rupture might guide diagnostic approaches to identify the patient at risk or drive therapeutic development to stagnate disease progression. A combination of various study designs is necessary for finding evidence to reach these ultimate goals: both longitudinal studies, investigating the in vivo relation between WSS and plaque progression (Kumar et al., 2018; Stone et al., 2018); in vitro assays, investigating the pathways underlying the response of endothelial cells to WSS; and cross-sectional investigations, proving the existence and applicability of these relations.

The variability in results between previous studies and ours might be due to different reasons. First, differences in geometry, size, and/or origin of the vascular bed might influence the nature of the relation between plaque composition and a hemodynamic parameter, such as WSS. This, in combination with the generally small sample sizes in this kind of studies, increases the likelihood of varying outcomes. Second, the degree of disease progression is likely to influence findings in cross-sectional setups. As plaque histology could only be obtained from subjects who are eligible for an endarterectomy procedure, the study population involved elderly patients with advanced disease. As relations between WSS and plaque composition is dynamic and disease stage-dependent, these relations could be less obvious in our study population. Third, the study setup might be of influence. Only one previous study compared TAWSS and OSI to histologically determined vulnerability features in four CEA samples (Kaazempur-Mofrad et al., 2004) and found no correlations. In all other cross-sectional studies, plaque composition was determined by in vivo imaging, or relative WSS levels were assumed based on axial location instead of calculations. Finally, we studied relative differences in WSS parameters, not absolute levels, in a cross-sectional setup. With time, technological improvements regarding higher in vivo imaging resolutions might allow patient-specific flow data and high-resolution compositional information to be obtained. This in combination with a larger study number would be required to analyze whether WSS parameters can be used as a predictor of plaque composition and ultimately rupture risk.

In conclusion, our image registration pipeline can match histology to patient-derived WSS metrics and can be used to investigate the relation between these WSS metrics and features of plaque vulnerability. We found relations between TAWSS and $\mathrm{NC}$ area and between TAWSS and macrophage area, which were in agreement with the literature. To our knowledge, our study is the first to show significant relations between OSI and features of human plaque vulnerability. Unlike the general assumption, low OSI areas did not always coincide with high TAWSS. In fact, areas exposed to both low OSI and low TAWSS showed significantly thicker caps. 


\section{DATA AVAILABILITY STATEMENT}

The datasets presented in this article are not readily available because the data is generated in a consortium. Approval by the consortium is needed before the data can be shared. Requests to access the datasets should be directed to k.vanderheiden@ erasmusmc.nl.

\section{ETHICS STATEMENT}

The studies involving human participants were reviewed and approved by the Institutional Medical Ethical Committee. The patients/participants provided their written informed consent to participate in this study.

\section{REFERENCES}

Ahmadi, A., Leipsic, J., Blankstein, R., Taylor, C., Hecht, H., Stone, G. W., et al. (2015). Do Plaques Rapidly Progress Prior to Myocardial Infarction? Circ. Res. 117, 99-104. doi:10.1161/CIRCRESAHA.117.305637

Antiga, L., Piccinelli, M., Botti, L., Ene-Iordache, B., Remuzzi, A., and Steinman, D. A. (2008). An Image-Based Modeling Framework for Patient-specific Computational Hemodynamics. Med. Biol. Eng. Comput. 46, 1097-1112. doi:10.1007/s11517-008-0420-1

Boekhoven, R. W., Lopata, R. G. P., van Sambeek, M. R., van de Vosse, F. N., and Rutten, M. C. M. (2013). A Novel Experimental Approach for ThreeDimensional Geometry Assessment of Calcified Human Stenotic Arteries In Vitro. Ultrasound Med. Biol. 39, 1875-1886. doi:10.1016/j.ultrasmedbio.2013. 03.019

Burke, A. P., Farb, A., Malcom, G. T., Liang, Y.-h., Smialek, J. E., and Virmani, R. (1999). Plaque Rupture and Sudden Death Related to Exertion in Men with Coronary Artery Disease. Jama 281, 921-926. doi:10.1001/jama.281.10.921

Cicha, I., Wörner, A., Urschel, K., Beronov, K., Goppelt-Struebe, M., Verhoeven, E., et al. (2011). Carotid Plaque Vulnerability. Stroke 42, 3502-3510. doi:10.1161/ strokeaha.111.627265

Cunningham, K. S., and Gotlieb, A. I. (2005). The Role of Shear Stress in the Pathogenesis of Atherosclerosis. Lab. Invest. 85, 9-23. doi:10.1038/labinvest. 3700215

Daugherty, A., Tall, A. R., Daemen, M. J. A. P., Falk, E., Fisher, E. A., GarcíaCardeña, G., et al. (2017). Recommendation on Design, Execution, and Reporting of Animal Atherosclerosis Studies: A Scientific Statement from the American Heart Association. Arterioscler Thromb. Vasc. Biol. 37, e131-e157. doi:10.1161/ATV.0000000000000062

de Weert, T. T., Cretier, S., Groen, H. C., Homburg, P., Cakir, H., Wentzel, J. J., et al. (2009). Atherosclerotic Plaque Surface Morphology in the Carotid Bifurcation Assessed with Multidetector Computed Tomography Angiography. Stroke 40, 1334-1340. doi:10.1161/STROKEAHA.108.538439

Dirksen, M. T., van der Wal, A. C., van den Berg, F. M., van der Loos, C. M., and Becker, A. E. (1998). Distribution of Inflammatory Cells in Atherosclerotic Plaques Relates to the Direction of Flow. Circulation 98, 2000-2003. doi:10. 1161/01.cir.98.19.2000

Eshtehardi, P., McDaniel, M. C., Suo, J., Dhawan, S. S., Timmins, L. H., Binongo, J. N. G., et al. (2012). Association of Coronary wall Shear Stress with Atherosclerotic Plaque burden, Composition, and Distribution in Patients with Coronary Artery Disease. Jaha. Assoc. 1, e002543. doi:10.1161/JAHA. 112.002543

Fagerberg, B., Ryndel, M., Kjelldahl, J., Akyürek, L. M., Rosengren, L., Karlström, L., et al. (2010). Differences in Lesion Severity and Cellular Composition between In Vivo Assessed Upstream and Downstream Sides of Human Symptomatic Carotid Atherosclerotic Plaques. J. Vasc. Res. 47, 221-230. doi:10.1159/000255965

\section{AUTHOR CONTRIBUTIONS}

$\mathrm{KH}$ and FG contributed to conception and design of the study. $\mathrm{AM}, \mathrm{KD}, \mathrm{DP}$, and KG performed the experiments and/or general analysis. SK and AM performed the computational modeling. $\mathrm{AM}, \mathrm{KH}, \mathrm{FG}$, and $\mathrm{AS}$ interpreted the data. $\mathrm{AM}, \mathrm{KH}$, and JW performed the statistical analysis. $\mathrm{KH}, \mathrm{AL}$, and $\mathrm{HV}$ organized the human carotid plaque samples. AM and $\mathrm{KH}$ wrote the first draft of the manuscript. All authors contributed to manuscript revision, read, and approved the submitted version.

\section{FUNDING}

$\mathrm{AM}$ and $\mathrm{KH}$ are funded by the Netherlands Heart Foundation (NHS2014T096).

Falk, E., Nakano, M., Bentzon, J. F., Finn, A. V., and Virmani, R. (2013). Update on Acute Coronary Syndromes: the Pathologists' View. Eur. Heart J. 34, 719-728. doi:10.1093/eurheartj/ehs411

Gallo, D., Steinman, D. A., and Morbiducci, U. (2016). Insights into the Colocalization of Magnitude-Based versus Direction-Based Indicators of Disturbed Shear at the Carotid Bifurcation. J. Biomech. 49, 2413-2419. doi:10.1016/j.jbiomech.2016.02.010

Gimbrone, M. A., and García-Cardeña, G. (2013). Vascular Endothelium, Hemodynamics, and the Pathobiology of Atherosclerosis. Cardiovasc. Pathol. 22 (1), 9-15. doi:10.1016/j.carpath.2012.06.006

Gnasso, A., Irace, C., Carallo, C., De Franceschi, M. S., Motti, C., Mattioli, P. L., et al. (1997). In Vivo Association between Low Wall Shear Stress and Plaque in Subjects with Asymmetrical Carotid Atherosclerosis. Stroke 28, 993-998. doi:10.1161/01.STR.28.5.993

Groen, H. C., Gijsen, F. J. H., van der Lugt, A., Ferguson, M. S., Hatsukami, T. S., van der Steen, A. F. W., et al. (2007). Plaque Rupture in the Carotid Artery Is Localized at the High Shear Stress Region. Stroke 38, 2379-2381. doi:10.1161/ STROKEAHA.107.484766

Groen, H. C., Simons, L., van den Bouwhuijsen, Q. J. A., Bosboom, E. M. H., Gijsen, F. J. H., Van Der Giessen, A. G., et al. (2010). MRI-based Quantification of Outflow Boundary Conditions for Computational Fluid Dynamics of Stenosed Human Carotid Arteries. J. Biomech. 43, 2332-2338. doi:10.1016/j.jbiomech. 2010.04.039

Kaazempur-Mofrad, M. R., Isasi, A. G., Younis, H. F., Chan, R. C., Hinton, D. P., Sukhova, G., et al. (2004). Characterization of the Atherosclerotic Carotid Bifurcation Using MRI, Finite Element Modeling, and Histology. Ann. Biomed. Eng. 32, 932-946. doi:10.1023/b:abme.0000032456.16097.e0

Ku, D. N., Giddens, D. P., Zarins, C. K., and Glagov, S. (1985). Pulsatile Flow and Atherosclerosis in the Human Carotid Bifurcation. Positive Correlation between Plaque Location and Low Oscillating Shear Stress. Arteriosclerosis 5, 293-302. doi:10.1161/01.ATV.5.3.293

Kumar, A., Thompson, E. W., Lefieux, A., Molony, D. S., Davis, E. L., Chand, N., et al. (2018). High Coronary Shear Stress in Patients with Coronary Artery Disease Predicts Myocardial Infarction. J. Am. Coll. Cardiol. 72, 1926-1935. doi:10.1016/j.jacc.2018.07.075

Kwak, B. R., Bäck, M., Bochaton-Piallat, M.-L., Caligiuri, G., Daemen, M. J. A. P., Davies, P. F., et al. (2014). Biomechanical Factors in Atherosclerosis: Mechanisms and Clinical Implications. Eur. Heart J. 35 (43), 3013-3020. doi:10.1093/eurheartj/ehu353

Ladisa, J. F., Bowers, M., Harmann, L., Prost, R., Doppalapudi, A. V., Mohyuddin, T., et al. (2010). Time-efficient Patient-specific Quantification of Regional Carotid Artery Fluid Dynamics and Spatial Correlation with Plaque burden. Med. Phys. 37, 784-792. doi:10.1118/1.3292631

Lee, M.-Y., Wu, C.-M., Yu, K.-H., Chu, C.-S., Lee, K.-T., Sheu, S.-H., et al. (2009). Association between Wall Shear Stress and Carotid Atherosclerosis in Patients with Never Treated Essential Hypertension. Am. J. Hypertens. 22, 705-710. doi:10.1038/ajh.2009.77 
Lee, S.-W., Antiga, L., Spence, J. D., and Steinman, D. A. (2008). Geometry of the Carotid Bifurcation Predicts its Exposure to Disturbed Flow. Stroke 39, 2341-2347. doi:10.1161/STROKEAHA.107.510644

Lovett, J. K., and Rothwell, P. M. (2003). Site of Carotid Plaque Ulceration in Relation to Direction of Blood Flow: an Angiographic and Pathological Study. Cerebrovasc. Dis. 16, 369-375. doi:10.1159/000072559

Lusis, A. J. (2000). Atherosclerosis. Nature 407, 233-241. doi:10.1038/ 35025203

Moerman, A. M., Dilba, K., Korteland, S., Poot, D. H. J., Klein, S., van der Lugt, A., et al. (2019). An MRI-Based Method to Register Patient-specific wall Shear Stress Data to Histology. PLoS One 14, e0217271. doi:10.1371/journal.pone.0217271

Park, J.-B., Choi, G., Chun, E. J., Kim, H. J., Park, J., Jung, J.-H., et al. (2016). Computational Fluid Dynamic Measures of wall Shear Stress Are Related to Coronary Lesion Characteristics. Heart 102, 1655-1661. doi:10.1136/heartjnl2016-309299

Richardson, P., Davies, M. J., and Born, G. V. R. (1989). Influence of Plaque Configuration and Stress Distribution on Fissuring of Coronary Atherosclerotic Plaques. The Lancet 334, 941-944. doi:10.1016/S0140-6736(89)90953-7

Ruifrok, A. C., and Johnston, D. A. (2001). Quantification of Histochemical Staining by Color Deconvolution. Anal. Quant. Cytol. Histol. 23, 291-299.

Samady, H., Eshtehardi, P., McDaniel, M. C., Suo, J., Dhawan, S. S., Maynard, C., et al. (2011). Coronary Artery wall Shear Stress Is Associated with Progression and Transformation of Atherosclerotic Plaque and Arterial Remodeling in Patients with Coronary Artery Disease. Circulation 124, 779-788. doi:10.1161/ CIRCULATIONAHA.111.021824

Schaar, J., Muller, J. E., Falk, E., Virmani, R., Fuster, V., Serruys, P. W., et al. (2004). Terminology for High-Risk and Vulnerable Coronary Artery Plaques. Eur. Heart J. 25, 1077-1082. doi:10.1016/j.ehj.2004.01.002

Schindelin, J., Arganda-Carreras, I., Frise, E., Kaynig, V., Longair, M., Pietzsch, T., et al. (2012). Fiji: An Open-Source Platform for Biological-Image Analysis. Nat. Methods 9, 676-682. doi:10.1038/nmeth.2019

Seo, T., Schachter, L. G., and Barakat, A. I. (2005). Computational Study of Fluid Mechanical Disturbance Induced by Endovascular Stents. Ann. Biomed. Eng. 33, 444-456. doi:10.1007/s10439-005-2499-y

Slager, C., Wentzel, J., Gijsen, F., Thury, A., van der Wal, A., Schaar, J., et al. (2005). The Role of Shear Stress in the Destabilization of Vulnerable Plaques and Related Therapeutic Implications. Nat. Rev. Cardiol. 2, 456-464. doi:10.1038/ncpcardio0298

Stone, P. H., Maehara, A., Coskun, A. U., Maynard, C. C., Zaromytidou, M., Siasos, G., et al. (2018). Role of Low Endothelial Shear Stress and Plaque Characteristics in the Prediction of Nonculprit Major Adverse Cardiac Events. JACC: Cardiovasc. Imaging 11, 462-471. doi:10.1016/j.jcmg.2017.01.031

Tuenter, A., Selwaness, M., Arias Lorza, A., Schuurbiers, J. C. H., Speelman, L., Cibis, M., et al. (2016). High Shear Stress Relates to Intraplaque Haemorrhage in Asymptomatic Carotid Plaques. Atherosclerosis 251, 348-354. doi:10.1016/j. atherosclerosis.2016.05.018

Vamsi Krishna, C., Chandran Suja, V., Watton, P. N., Arakeri, J. H., and Gundiah, N. (2020). Shear Stress Rosettes Capture the Complex Flow Physics in Diseased Arteries. J. Biomech. 104, 109721. doi:10.1016/j.jbiomech.2020.109721

van Ooij, P., Cibis, M., Rowland, E. M., Vernooij, M. W., van der Lugt, A., Weinberg, P. D., et al. (2018). Spatial Correlations between MRI-Derived wall Shear Stress and Vessel wall Thickness in the Carotid Bifurcation. Eur. Radiol. Exp. 2, 27. doi:10.1186/s41747-018-0058-1

Vergallo, R., Papafaklis, M. I., Yonetsu, T., Bourantas, C. V., Andreou, I., Wang, Z., et al. (2014). Endothelial Shear Stress and Coronary Plaque Characteristics in Humans. Circ. Cardiovasc. Imaging 7, 905-911. doi:10.1161/circimaging.114.001932

Virmani, R., Ladich, E. R., Burke, A. P., and Kolodgie, F. D. (2006b). Histopathology of Carotid Atherosclerotic Disease. Neurosurgery 59, S3219-S3-227. doi:10.1227/01.NEU.0000239895.00373.E4
Virmani, R., Burke, A. P., Farb, A., and Kolodgie, F. D. (2006a). Vulnerable Plaque Symposium, Boston, Massachusetts, USA, October 2003. J. Am. Coll. Cardiol. 47, C1-C103. doi:10.1016/j.jacc.2005.06.090

Virmani, R., Kolodgie, F. D., Burke, A. P., Farb, A., and Schwartz, S. M. (2000). Lessons from Sudden Coronary Death. Atvb 20, 1262-1275. doi:10.1161/01.atv. 20.5.1262

Wentzel, J. J., Chatzizisis, Y. S., Gijsen, F. J. H., Giannoglou, G. D., Feldman, C. L., and Stone, P. H. (2012). Endothelial Shear Stress in the Evolution of Coronary Atherosclerotic Plaque and Vascular Remodelling: Current Understanding and Remaining Questions. Cardiovasc. Res. 96 (2), 234-243. doi:10.1093/cvr/cvs217

Wentzel, J. J., Janssen, E., Vos, J., Schuurbiers, J. C. H., Krams, R., Serruys, P. W., et al. (2003). Extension of Increased Atherosclerotic wall Thickness into High Shear Stress Regions Is Associated with Loss of Compensatory Remodeling. Circulation 108, 17-23. doi:10.1161/01.CIR.0000078637.21322.D3

Wentzel, J. J., Schuurbiers, J. C. H., Gonzalo Lopez, N., Gijsen, F. J. H., van der Giessen, A. G., Groen, H. C., et al. (2013). In Vivo assessment of the Relationship between Shear Stress and Necrotic Core in Early and Advanced Coronary Artery Disease. EuroIntervention 9, 989-995. doi:10. 4244/EIJV9I8A165

Winkel, L. C., Hoogendoorn, A., Xing, R., Wentzel, J. J., and Van der Heiden, K. (2015). Animal Models of Surgically Manipulated Flow Velocities to Study Shear Stress-Induced Atherosclerosis. Atherosclerosis 241, 100-110. doi:10. 1016/j.atherosclerosis.2015.04.796

Xu, P., Liu, X., Zhang, H., Ghista, D., Zhang, D., Shi, C., et al. (2018). Assessment of Boundary Conditions for CFD Simulation in Human Carotid Artery. Biomech. Model. Mechanobiol. 17, 1581-1597. doi:10.1007/s10237-018-1045-4

Yamamoto, E., Thondapu, V., Poon, E., Sugiyama, T., Fracassi, F., Dijkstra, J., et al. (2019). Endothelial Shear Stress and Plaque Erosion. JACC: Cardiovasc. Imaging 12, 374-375. doi:10.1016/j.jcmg.2018.07.024

Yang, C., Canton, G., Yuan, C., Ferguson, M., Hatsukami, T. S., and Tang, D. (2010). Advanced Human Carotid Plaque Progression Correlates Positively with Flow Shear Stress Using Follow-Up Scan Data: an In Vivo MRI MultiPatient 3D FSI Study. J. Biomech. 43, 2530-2538. doi:10.1016/j.jbiomech.2010. 05.018

Yushkevich, P. A., Piven, J., Hazlett, H. C., Gerig, G., Ho, S., Gee, J. C., et al. (2006). User-guided 3D Active Contour Segmentation of Anatomical Structures: Significantly Improved Efficiency and Reliability. Neuroimage 31, 1116-1128. doi:10.1016/j.neuroimage.2006.01.015

Conflict of Interest: The authors declare that the research was conducted in the absence of any commercial or financial relationships that could be construed as a potential conflict of interest.

Publisher's Note: All claims expressed in this article are solely those of the authors and do not necessarily represent those of their affiliated organizations, or those of the publisher, the editors and the reviewers. Any product that may be evaluated in this article, or claim that may be made by its manufacturer, is not guaranteed or endorsed by the publisher.

Copyright $\odot 2022$ Moerman, Korteland, Dilba, van Gaalen, Poot, van Der Lugt, Verhagen, Wentzel, van Der Steen, Gijsen and Van der Heiden. This is an openaccess article distributed under the terms of the Creative Commons Attribution License (CC BY). The use, distribution or reproduction in other forums is permitted, provided the original author(s) and the copyright owner(s) are credited and that the original publication in this journal is cited, in accordance with accepted academic practice. No use, distribution or reproduction is permitted which does not comply with these terms. 